\title{
Zum privaten Werkvertrag im Rahmen der neusumerischen handwerklichen Produktion
}

Der handwerklichen Produktion als der zweiten wichtigen Produktionsform nach der Landwirtschaft kam im Rahmen der Ökonomie des neusumerischen Reiches von Ur eine große Bedeutung zu. Zum einen diente das Handwerk der Herstellung von Geräten, vor allem für die landwirtschaftliche Produktion, zum anderen war es von entscheidender Bedeutung bei der Befriedigung von Luxusbedürfnissen der mit Palast und Tempel verbundenen Oberschicht. Hinzu kamen wichtige Anwer. dungsbereiche außerhalb des Ackerbaus und der Luxusgüterherstellung, wie $\mathrm{z}$. $\mathrm{F}$ Militär-, Transport- und Bauwesen sowie die Schiffahrt.

Die dominierende Stellung des Staates in der Ur III-Ökonomie führte zu einer Kor:zentration eines bedeutenden Teils der handwerklichen Produktion im Bereich de? Palast- und Tempelwirtschaft. Dabei ist allerdings zu beachten, daß das Handwer: im Dienst der verschiedenen Verwaltungs- und Wirtschaftseinheiten in den einzenen Städten und Provinzen des neusumerischen Reiches nicht nach einem einheitlchen Prinzip organisiert war. Dies gründet sich vor allem auf die unterschiedlichic ökonomische Stellung dieser Städte im Wirtschaftsgefüge des Reiches, was unter anderem auch in engem Zusammenhang mit ihrer jeweiligen politischen und kultischen Bedeutung sowie den verschiedentlich wirkenden Traditionen zu sehen ist. ${ }^{1}$

1 Vgl. dazu im einzelnen H. Neumann, Handwerk in Mesopotamien. Untersuchungen "u seiner Organisation in der Zeit der III. Dynastie von Ur, Berlin, 1987. (SGKAO 19). - itl den im vorliegenden Beitrag verwendeten Abkürzungen vgl. W, von Soden, Akkadisch 's Handwörterbuch, Wiesbaden 1959-1981 (AHw) und ergänzend Keilschriftbibliograplje 47, Or.NS 55 [1986] 1*-5*. Zusätzliche Abkürzungen sind: AAS = J.-P. Grégoire, Archives administratives sumériennes, Paris 1970; BBVO = Berliner Beiträge zum Vorderen Orient, Berlin; CU = Codex Ur-Nammu; FAOS = Freiburger Altorientalische Studien, (Wiesbaden-) Stuttgart; $\mathrm{MC}=$ Mesopotamian Civilizations, Winona Lake; MCSA = Mesopotamia. $\mathrm{Co-}$ penhagen Studies in Assyriology; NRVN I = M. Çĭg - H. Kızılyay, Neusumerische Rechtsund Verwaltungsurkunden aus Nippur I, Ankara 1965; OBO = Orbis Biblicus et Orientalis, Fribourg - Göttingen; PSD = The Sumerian Dictionary of the University Museum of the University of Pennsylvania, Philadelphia; Rochester $=$ M. Sigrist, Documents from Tablet Collections in Rochester, New York, Bethesda 1991; SGKAO = Schriften zur Geschichte und Kultur des Alten Orients, Berlin; SNSAT = T. Gomi - S. Sato, Selected Neo- 
Ein Teil der Produktion erfolgte in staatlichen Werkstätten, die organisatorisch und verwaltungsmäßig in die Wirtschaftseinheiten der Palast- und Tempelökonomie eingebunden waren. So läßt sich in Ur, der Hauptstadt des neusumerischen Reiches, ein zentrales ,Handwerkerhaus' nachweisen, das in erster Linie für die Luxusgüterherstelung für den Königshof und die Hauptheiligtümer zuständig war. Man geht gewiß nicht ehl in der Annahme, wenn man die Organisation des Handwerks im Rahmen eines terartigen ,Handwerkerhauses' mit der Rolle von Ur als Königsresidenz in Verbindung ringt. ${ }^{2}$ In der Provinz Lagaš war ein Teil der handwerklichen Produktion in die Adninistration einzelner Tempelwirtschaften einbezogen, und es lassen sich darüber hin: us Handwerker auch in Webereien, Mühlen und Werften nachweisen. Die Kontrolle ïber die Produktion sowie die Koordination der einzelnen Teilbereiche des Handwerks urfolgte dabei durch die zentrale Provinzverwaltung. Wahrscheinlich führte die staatliche Administration damit traditionelle Organisationsprinzipien der Ökonomie des Stadtstaates von Lagaš weiter und machte sich diese zunutze. ${ }^{3}$ In Umma wurden die virtschaftlichen Aktivitäten der Provinz zentral organisiert, ohne sich allzusehr auf einzelne Tempelwirtschaften zu stützen. Entscheidende Funktionen scheinen dabei von venigen Familien ausgeübt worden zu sein. ${ }^{4}$

Die soziale Stellung der im Rahmen der staatlichen Wirtschaft tätigen Handverker war differenziert. Ein Teil der Arbeitskräfte gehörte zum unmittelbaren P:rsonal der Palast- und Tempelwirtschaft, was durch die Tatsache gekennzcichnet wird, daß sie Empfänger von Rationen und Gersteversorgungen waren. Darüber hinaus ergänzte die staatliche Verwaltung ihr eigenes Arbeitsk a aftepotential durch die zusätzliche Miete von qualifizierten Handwerkern, die $\mathrm{fi}$ ihre Dienste einen Lohn ausgezahlt bekamen. Ferner waren für die Palastu: d Tempelwirtschaft auch Handwerker tätig, die Auftragsarbeiten ausführten, oi ne jedoch in das Personal der staatlichen Wirtschaft eingegliedert zu sein. Lc zteres läßt sich für verschiedene Handwerkszweige feststellen, so $\mathrm{z}$. B. für di. Schmiede in Lagaš $\breve{5}^{5}$, $\mathrm{Umma}^{6}$ und Nippur ${ }^{7}$, die Goldschmiede ${ }^{8}$ und Stein-

sumerian Administrative Texts from the British Museum, Shiba 1990; UAVA = Untersuhungen zur Assyriologie und Vorderasiatischen Archäologie, Berlin-New York; Um. = F. Yıldız - T Gomi, Die Umma-Texte aus den Archäologischen Museen zu Istanbul III (Nr. 601-2300), Bethesda 1993; bei Datenangaben: AS = Amar-Su'ena; Dne. = Datum nicht

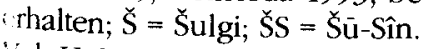

igl. H. Neumann, SGKAO 19, 35-71.

Fbenda $92-97$.

thenda 113-143.

Ygl. ebenda 97-106; zur Organisation des Schmiedehandwerks in der Provinz Lagaš vgl. jetzt auch B. Lafont, Les forgerons Sumériens de la ville de Girsu, in: J. des Courtils - J.Ch. Moretti - F. Planet (Hrsg.), De Anatolia Antiqua. Eski Anadolu, I., Traveaux et recherches de l'Institut Français d'Études Anatoliennes, Paris 1991, 119-129.

Vgl. H. Neumann, SGKAO 19, 113-124.

Vgl. R. L. Zettler, The Ur III Temple of Inanna at Nippur. The Operation and Organization of Urban Religious Institutions in Mesopotamia in the Late Third Millennium B. C., Berlin 1992 (BBVO 11), 227-230.

Vgl. H. Neumann, SGK AO 19, 111f. 125-127; R. L. Zettler, BBVO 11, 231. 
schneider ${ }^{9}$, aber auch für den Bereich der Leder- und Filzverarbeitung ${ }^{10}$ und für die Töpferei. ${ }^{11}$

Die Miete von qualifizierten Arbeitskräften gegen Zahlung eines Lohnes sowie die Praxis der Auftragsvergabe für handwerkliche Tätigkeiten an Bereiche außerhalb der staatlichen Verwaltung setzten die Existenz eines freien, nicht in die Palast- und Tempelwirtschaft eingebundenen Handwerks voraus. Insbesondere für einige Schmiede der Provinz Lagaš, die ihre Tätigkeit im Rahmen der staatlicher: Wirtschaft wahrscheinlich als freie Handwerker ausübten, läßt sich mit guten Gründen vermuten, daß sie eigene Werkstätten betrieben und darin auch Skla ven beschäftigten. ${ }^{12}$

Nun ist über die handwerkliche Produktion außerhalb der Palast- und Tempelwirtschaft kaum etwas bekannt. Dies ist im wesentlichen darin begründer, daß uns in erster Linie Urkunden der staatlichen Verwaltung überliefert sinc, während private Rechtsurkunden nur in relativ geringer Anzahl vorliegen. Für die Kennzeichnung der handwerklichen Produktion im privaten Bereich wären dabei vor allem Werkverträge, die handwerkliche Arbeiten zum Gegenstand haben, von Interesse. ${ }^{13}$ Daß uns eindeutige Verträge dieser Art zwischen Privatpersone? bislang fehlen, ${ }^{14}$ liegt zusätzlich aber auch daran, daß schriftlich ausgefertigle Werkverträge nach Ausführung des Werkes gewiß zum Teil wieder vernicht tt wurden. Ferner wird man auch damit zu rechnen haben, daß Werkverträge nic it in jedem Fall schriftlich fixiert wurden, wobei in diesem Zusammenhang hi:sichtlich der vereinbarten handwerklichen Arbeiten vielleicht auch die Art dos

${ }^{9}$ Vgl. etwa Rochester 238, wonach der Steinschneider ( $\mathrm{zadim}$ ) Huwawa $2^{1 / 2}{ }_{2}$ Minen Kı)fer von einem gewissen Ur-Lama in Empfang nahm; der Vermerk gi 4 $_{4}$-gi 4 -d a m (Z. 5) is ist zurückzugeben" könnte auf ein Darlehen oder aber auf einen Werkvertrag (mit c er staatlichen Verwaltung?) schließen lassen (nicht sicher, ob Verwaltungstext oder Recl isurkunde).

${ }^{10}$ Vgl. H. Neumann, SGKAO 19, 106-109 sowie den Text OrAnt. 19, 100 (A 5186), won

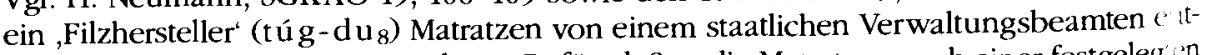
gegennahm, wohl um sie zu säubern. Dafür, daß er die Matratzen nach einer festgeleg en Frist wieder zurückbringen werde, hat er den Eid geleistet. Dazu P. Steinkeller, ebenda 74 zutreffend: "The use of a promissory oath allows us to classify this text as a legal do utment, more specifically, a promise to perform work-service during a specified period of time. As such, the present text can be compared to the OB 'Werkverträge".

" Vgl. P. Steinkeller, The Organization of Crafts in Third Millennium Babylonia: The Cast of Potters, AoF 23 [1996] 232-253.

${ }^{12}$ Vgl. dazu H. Neumann, SGKAO 19, $152 \mathrm{f}$.

13 Zum Werkvertrag vgl. M. San Nicolò, Beiträge zur Rechtsgeschichte im Bereich der kieitschriftlichen Rechtsquellen, Oslo 1931, 248-253; ders., Der neubabylonische Lehrveriag in rechtsvergleichender Betrachtung, München 1950 (SBAW 1950/3), 21 f.; E. Pritsch, Zur juristischen Bedeutung der šubanti-Formel, Bonner Biblische Beiträge 1 [1950] 184 mit Anm. 81; H. Petschow, ZA 57 [1965] 164-166.

${ }^{14}$ Dies gilt im übrigen auch für private Lehrverträge; hinsichtlich der geringen Anzahl derartiger Urkunden im Rahmen der keilschriftlichen Überlieferung insgesamt meint A. Falkenstein, ZSSR 68 [1951] 534, daß dies mit der Tatsache in Verbindung zu bringen sei, daß "das Handwerk überwiegend, wenn auch nicht ausschließlich, Familienbetrieb“ war. 
auszuführenden Werkes sowie der Wert der dafür bereitgestellten Materialien eine Rolle gespielt haben.

Daß Werkverträge zwischen Privatpersonen auch in der Ur III-Zeit abgeschlossen wurden, zeigt die aus Girsu stammende Gerichtsurkunde NG II 131 (= (TT II 3538), in der es um einen Prozeß wegen der Nichterfüllung eines Werkvertrages geht: ${ }^{15}$

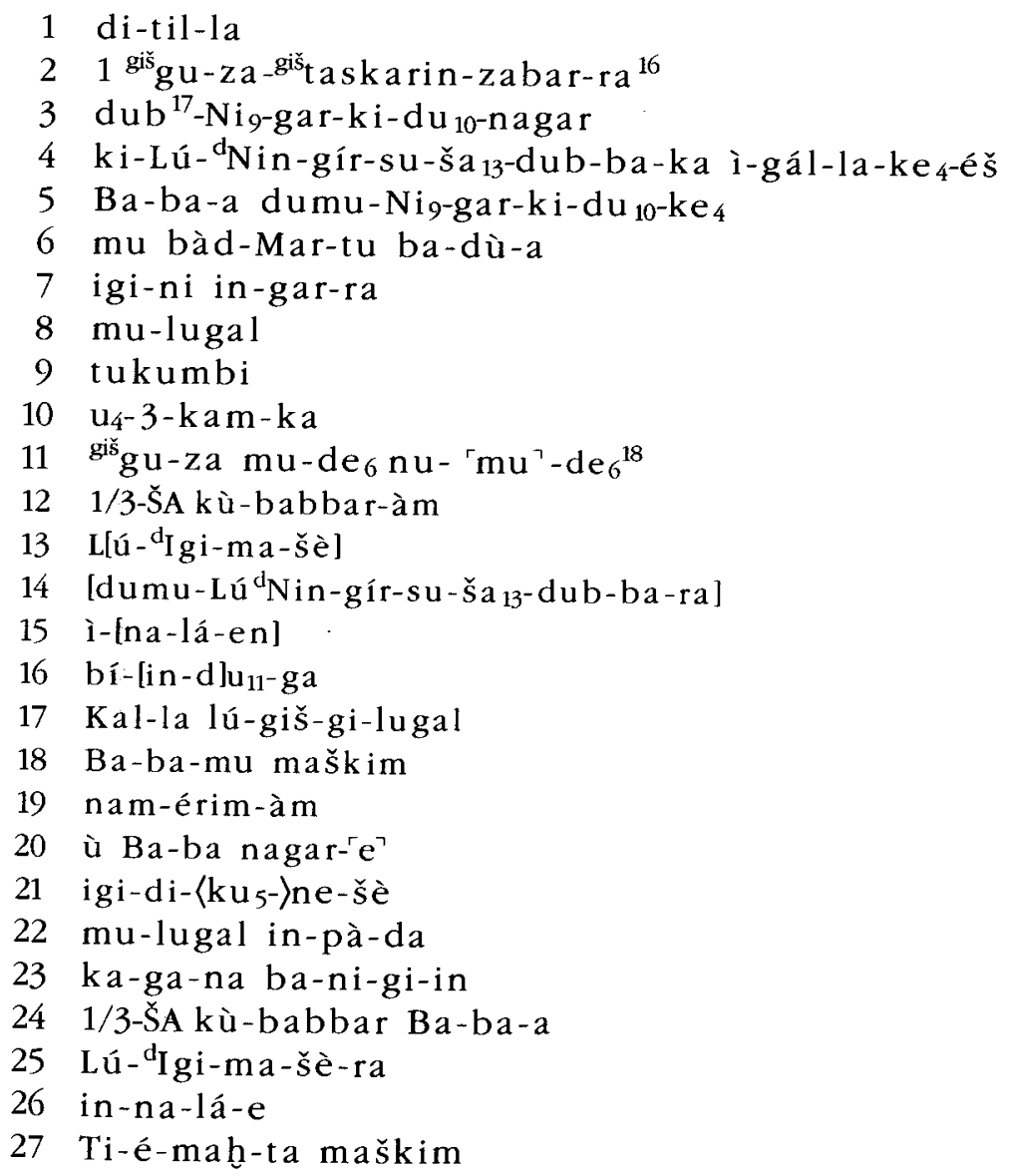

${ }^{15} \mathrm{Vgl}$. A. Falkenstein, NG II, 222-225; zum Text vgl. auch S. O'he, ASJ 2 [1980] 126-128; H. Neumann, SGKAO 19, $153 \mathrm{f}$.

17 Zur Lesung der Zeile vgl. A. Falkenstein, NG III, 9.

Neben $\mathrm{dub}=t / t u p p u$,Tafel, Urkunde“" wäre auch die Lesung kiš ib = kunukku in der Bedeutung ,gesiegelte Urkunde“ möglich; zur Identität der Zeichen in der Ur III-Zeit vgl.

18 A. Falkenstein, NG III, 129. Vgl. auch C. Wilcke, ZA 78 [1988] 29 mit Anm. 101.

Zu der hier anzusetzenden bamtu-Basis de 6 von DU ,tragen, bringen“ vgl. J. Krecher, ZA 63 [1973] 223 zu II 3; M.-L. Thomsen, The Sumerian Language. An Introduction to its History and Grammatical Structure, Kopenhagen 1984 (MCSA 10), 133. 

28 Lú-ď́śra
29 L[ú-din]gir-r[a di-ku 5$]-b i-m e$

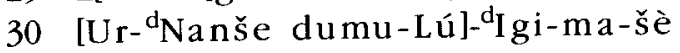
31 [R]a [dumu-Lú-i]g[i-s]a 6 -[s]a6
32 [Lu]gal-síg-su 13
33 lú-mar-za ki-ba gub-ba-me
$34 \mathrm{mu}^{\mathrm{d}} \mathfrak{S} u^{\mathrm{d}}{ }^{\mathrm{d}} \operatorname{Sin}$ lugal-e na-rú-a-maḩ ${ }^{\mathrm{d}}$ En-líl-lá ${ }^{\mathrm{d}} \mathrm{Nin-líl-lá}$ mu-ne-rú

\section{„Abgeschlossene Rechtssache:}

Daß, weil sich über einen Sessel aus Buchsbaumholz (und) Bronze (als das zu liefernde Werk) die Tafel des Nigarkidu, des Tischlers, bei Lu-Ningirsu, dem ,Rechnungsführer ${ }^{19}$, befindet, Baba, der Sohn des Nigarkidu, im Jahre ,Die Martu-Mauer wurde gebaut' (= SS 4) erschienen ist (und): „Beim König! Wenn ich in drei Tagen den Sessel bringe, (ist es gut); wenn ich ihn nicht bringe, ${ }^{20}$ werde ich $1 / 3$ (Mine) Silber dem L[u-Igimaše], [dem Sohn des Lu-Ningirsu, des ,Rechnungsführers'], z[al len]!“, er[klä]rt hat, haben Kalla, der ... des Königs, ${ }^{21}$ (und) Babamu, der (dam:lige) Kommissär, beschworen ${ }^{22}$ und Baba, der Tischler, hat, daß er vor den Rick:tern den Eid beim König geschworen hat, mit seiner Aussage bestätigt. ${ }^{23} 1 / 3$ (Minc) Silber wird Baba dem Lu-Igimaše (wegen Nichterfüllung des Werkvertrages) zal:len.

Ti'emahta (war dabei) Kommissär. Lu-Šara (und) L[udin]gir[a] waren die dafır (zuständigen) [Richter].

[Ur-Nanše, der Sohn des Lu]-Igimaše, [R]a der [Sohn des Lui]g[is]a[s]a, (un ) [Lu]galsigsu waren die marza-Leute ${ }^{24}$, die dabeistanden.

$\mathrm{Zu} \mathrm{ša} \mathrm{a}_{13}$-dub-ba = šandabakku, Rechnungsführer“ o. ä. vgl. A. Falkenstein, NG III, 159 (r it Literatur); J.-P. Grégoire, AAS, 44 Anm. 17 (mit Literatur); AHw 1162b; R. K. Englund, BB O 10, $60 \mathrm{f}$. („Hauptbuchführer"). Die von W. Sallaberger, Der kultische Kalender der Ur IIl-Z: it, Teil 1, Berlin-New York 1993 (UAVA 7/1), 168 vorgeschlagene Übersetzung „Feld-Schätz: $\mathrm{f}^{\text {" }}$ scheint mir zu eng gefaßt.

20 Zur Formulierung des mit tuku mbi „wenn“ eingeführten Konditionalsatzes vgl. A. I itkenstein, NG II, 224f. zu Z. 11; J. Black, ASJ 17 [1995] 38f.

21 Die Funktionsbezeichnung(?) lú -giš-gi ist mir unklar; vgl. A. Falkenstein, NG III, 35 („Bote"“); \. W. Sjöberg, ZA 65 [1975] 186f. (Z. 87) und 227 (lú-giš-gi [und mí-giš-gi] ohne Übersetzung).

22 Zur vorliegenden Konstruktion des (assertorischen) Eides vgl. D. O. Edzard, in: Sumerological Studies in Honor of Thorkild Jacobsen on his Seventieth Birthday June 7, 1974, (hicago-London 1976 (AS 20), 89. Zum Eidformular vgl. jetzt auch P. Steinkeller, Sale Documents of the Ur-III-Period, Stuttgart 1989 (FAOS 17), 75-77.

23 Zur Wendung ka(-g)-...-a gi-(n) vgl. J. Krecher, ZA 69 [1979] 1-3: ,in der Aussage des ... festmachen/verankern" $={ }_{\text {" }}$ (den Sachverhalt $)$ durch eine entsprechende Aussage bestätigen."

${ }^{24} \mathrm{Zu}$ den lú-ma r-za, von A. Falkenstein, NG I, 54-58 als eine Art von ,Publizitätszeugen` gedeutet, vgl. auch $\mathrm{S}$. O'he, The Terms Lú ki-ba gub-ba and Lú-mar-za ki-ba gub-ba in the Ur III Texts, ASJ 2 [1980] 126-140. 
Jahr: Šù-Sîn, der König, hat die Hohe Stele für Enlil (und) Ninlil errichtet = ك̌S 6)."

Nach der vorliegenden Prozeßurkunde hat sich der Tischler Nigarkidu zur Lieerung eines Sessels aus Buchsbaumholz und Bronze gegenüber dem ,Rechnungsführer' Lu-Ningirsu ${ }^{25}$ verpflichtet, ohne daß der nagar diese Verpflichtung iedoch zu Lebzeiten erfüllen konnte. Der Sohn des Nigarkidu, Baba, der wie sein Vater den Beruf eines Tischlers ausübte, sagte daraufhin die Erfüllung des Werkvertrages innerhalb von drei Tagen zu und verpflichtete sich, bei eventueller Nichterfüllung zur Zahlung eines Betrages von $1 / 3$ Mine Silber an Lu-Igimaše, den Sohn des Lu-Ningirsu. ${ }^{26} \mathrm{Baba}$ hatte somit die Verbindlichkeiten seines verstorbenen Vaters übernommen, ${ }^{27}$ ohne sie dann jedoch - wie der Prozeßverlauf zeigt - tatsächlich erfüllt zu haben. Da nach zwei Jahren die Lieferung immer noch nicht erfolgt war, wurde der Handwerker (gewiß nach der von Lu-Igimaše erhobenen Klage) dazu verurteilt, die das gelieferte Material kompensierende und wohl auch eine Verzögerungsbuße enthaltende Zahlung ${ }^{28}$ an Lu-Igimaše zu leisten.

Grundlage für die Lieferungsverpflichtung des Baba war der schriftlich fixierte verkvertrag seines Vaters Nigarkidu (Z. 3). Die entsprechende Tafel, die den Mavrialempfang und die Lieferungszusage beurkundet haben dürfte, ${ }^{29}$ befand sich in Besitz des Auftraggebers (Z. 4) und diente dessen Sohn als Nachweis für die r:och ausstehende Erfüllung der vom Tischler eingegangenen Verpflichtung. BeI : erkenswert ist, daß es sich bei dem Gegenstand des Werkvertrages um die Hersellung und Lieferung eines relativ wertvollen handwerklichen Produktes hancilte, was die Tätigkeit der Handwerker im Bereich der Luxusgüterproduktion für ien privaten Bedarf belegt ${ }^{30}$

In den vorliegenden Zusammenhang gehört auch der 1990 von T. Gomi und $\$$ Sato (leider nur in Umschrift) publizierte und aus Umma stammende Text S ISAT 535, in dem ebenfalls auf einen Werkvertrag verwiesen wird (Z. 1-5), der e'ne handwerkliche Arbeit zum Gegenstand hat: ${ }^{31}$

25. Zum ša ${ }_{13}$-du b-ba Lu-Ningirsu vgl. etwa noch SNSAT 260 Vs. III 20, Rs. II 1 ( $\breve{S} 32-34$ ); HSS IV 7 II 27 ( $\check{S} 48$ ).

${ }^{26}$ Zu dem Sohn des Lu-Ningirsu, dem ga la Lu-Igimaše, vgl. A. Falkenstein, NG I, 49 Nr. 40 (zu NG II 12 [= RTC 288], 19 [Š 32] und 135 [= RTC 292], 7 [AS 1]).

$27 \mathrm{Vgl}$. A. Falkenstein, NG II, 224. Zum erbrechtlichen Hintergrund vgl. auch H. Petschow, ZSSR 75 [1958] 387,

${ }^{28}$ Vgl. A. Falkenstein, NG II, 224.

2) Zum Inhalt des Werkvertrages vgl. ebenda zu Z. 3.

${ }^{30} \mathrm{Vgl}$. dazu noch die bei H. Neumann, SGKAO 19, 154 Anm. 877 zitierten Belege sowie das Kapitel „Die soziale Stellung der Handwerker nach Gerichts- und privaten Rechtsurkunden“ ebenda 155-157.

31 Zum Text vgl. auch H. Limet, in: M. E. Cohen - D. C. Snell - D. B. Weisberg (Hrsg.), The Tablet and the Scroll. Near Eastern Studies in Honor of William W. Hallo, Bethesda 1993, 142. Der Text wurde freundlicherweise von M. J. Geller kollationiert (vgl. Z. 4, 22f., 25 ). 


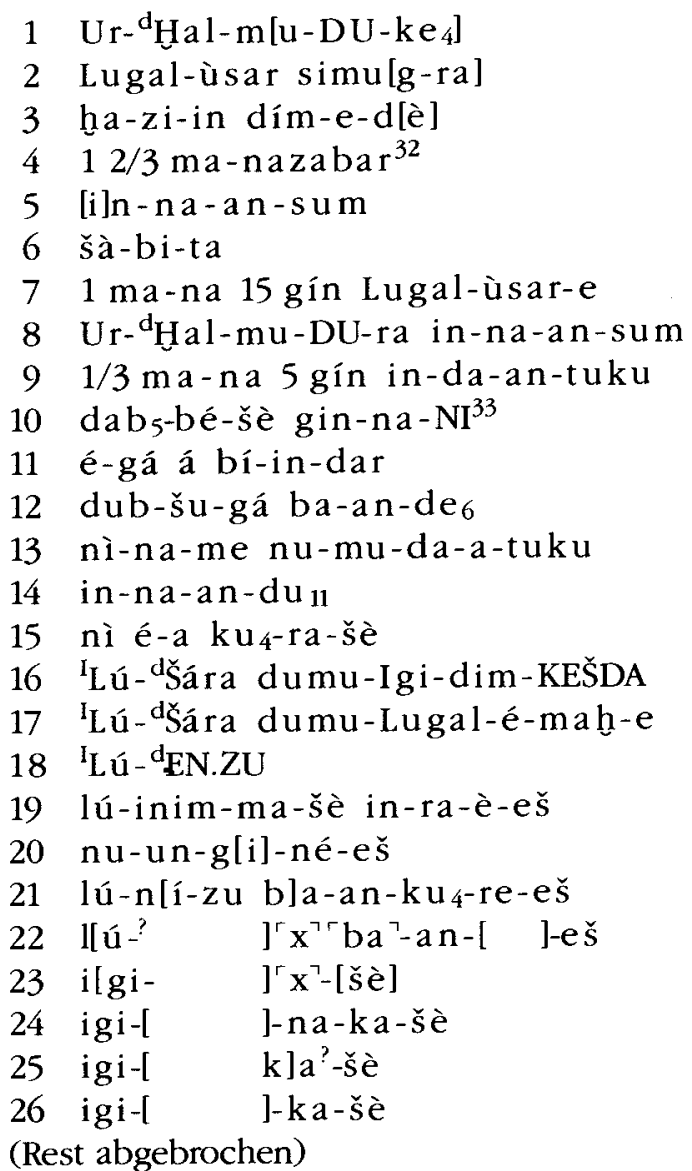

„Ur-Halm[u-DU] hat [dem] Lugalusar, [dem] Schmie[d], [u]m eine Axt herzustelk $n$, $12 / 3$ Mine Bronze gegeben. Davon hat 1 Mine 15 Sekel Lugalusar dem Ur-Halmu-1 U (zurück)gegeben. ${ }^{34} 1 / 3$ Mine 5 Sekel hat er (= Ur-Halmu-DU) (somit) bei ihm gut ${ }^{35}$ ${ }_{n}$ Als er kam, um es ( = die Bronze) zu nehmen, hat er es sich in meinem Haus se-

${ }^{32}$ Die Lesung zabar (UD. KA. BAR) „Bronze" wurde von M. J. Geller durch Kollation bestätigt. Unzutreffend daher PSD $\mathrm{A}_{2} 50 \mathrm{~b}$ "copper“. Es handelt sich also bei der hier dokumentierten Materiallieferung um einzuschmelzendes Metall.

33 -NI ist in dem vorliegenden Fall einer pronominalen Konjugation mit J. Krecher, ASI 15 [1993] 96-98 vielleicht als - né, die Postposition des Lokativ-Terminativs (Direktiv) enthaltend, aufzufassen.

${ }^{34}$ Gemeint ist das Gewicht des hergestellten Produkts, also der aus Bronze gefertigten Axt, die zwecks Ermittlung der Menge des verbrauchten Materials abgewogen wurde, so daß sich daraus auch das Gewicht des zurückzugebenden Metalls ergab.

${ }^{35}$ Hier handelt es sich um die für den neusumerischen Verpflichtungsschein typische Formulierung; vgl. A. Falkenstein, NG I, 119 mit Anm. 5; H. Lutzmann, Die neusumerischen 
waltsam angeeignet ${ }^{36}$ (und) die ,Tafel ${ }^{37}$ meiner Hand ${ }^{38}$ hat er davongetragen. ${ }^{39}$ Vichts hast du (noch) bei mir gut! ${ }^{40 "}$ hat er (= Lugalusar) ihm erklärt. ${ }^{41}$ Bezüglich der Sache, daß in das Haus eingedrungen worden ist, sind Lu-Sara, der Sohn des 'gidim-KEŠDA, Lu-Šara, der Sohn des Lugalemahe (und) Lu-EN.ZU als Zeugen aufzetreten; ${ }^{42}$ sie haben es nicht be[st]ätigt. ${ }^{43}$ Sie wurden zu ,Übelt[ätern'] erklärt. ${ }^{44}$

Schuldurkunden, Teil I: Einleitung und systematische Darstellung, Diss. Friedrich-Alexander-Universität Erlangen-Nürnberg 1976, $13 \mathrm{f}$. in - da - a n-t u k u nimmt im vorliegenden Fall wohl auch auf die nach erfolgter Lieferung des Werkes schriftlich ausgefertigte Schuldurkunde über das restliche (zurückzugebende) Material Bezug (vgl. unten Anm. 38 zu dub-šu-gá).

${ }^{3}$ Zu á-dar vgl. A. Falkenstein, NG II, 110 zu Z. 5; ders., NG III, 90 ( zu Unrecht zurückhalten, beschlagnahmen"); R. K. Englund, BBVO 10, 41 mit Anm. 143 ("in ... Gewalt nehmen“); PSD $\mathrm{A}_{2} 50 \mathrm{~b}$,to cheat (somebody) out of one's right', 'to seize illegally', 'to confiscate', 'to lay an embargoon (something)' [a legal phrase]"). Gemeint ist in vorliegender Urkunde die unrechtmäßige und wohl auch gewaltsam erfolgte Beschlagnahme der ausstehenden Materialmenge (ohne Einigung mit dem Schuldner), wie bereits das mit á-dar korrespondierende akkadische Formular ina emúqim dâșum zeigt (vgl. PSD $A_{2}$ 51a mit Verweis auf AbB IV 69, 25). Die Unrechtmäßigkeit der Handlung wird zudem dadurch unterstrichen, daß der Gläubiger auch die Schuldurkunde nach erfolgter Beschlagnahme dem Schuldner nicht ausgehändigt hatte; s. dazu weiter unten.

Vgl. dazu oben Anm. 17.

dub-šu-gá "Tafel meiner Hand“ bezeichnet gewiß die vom Schuldner (= Lugalusar) zugunsten des Gläubigers ( = Ur-Halmu-DU) ausgefertigte Schuldurkunde. Vgl. auch oben Anm. 35.

3u ba(-an)-de 6 „er hat an sich genommen/davongetragen“ vgl. A. Falkenstein, NG III, 169; J. Krecher, ZA 63 [1973] 223 zu II 3. Anders, jedoch unter sachlichen Gesichtspunkten sehr unwahrscheinlich, H. Limet, in: The Tablet and the Scroll, 142: "il a placé la tablette(?) dans ma main". Genau das Gegenteil dürfte richtig sein; s. dazu oben Anm. 36 und weiter unten.

40 Gegen H. Limet, in: The Tablet and the Scroll, 142 ("je ne lui dois plus rien") liegt bei numu-da-a-tuku die 2. Prs. Sg. vor (zum Wechsel des Subjektzeichens -e- $>-\mathbf{a}$ - nach vorausgehenden - da - vgl. M.-L. Thomsen, MCSA 10, 149f. Anm. 45).

${ }^{41}$ Die wörtliche Rede (des Lugalusar) enthält im ersten Teil die Schilderung des unrechtmäßigen Handelns des Ur-Halmu-DU in der 3. Prs. Sg. (Z. 10-12), im zweiten Teil die direkt an den Gläubiger in der Folge der Tat gerichtete Erklärung (Z. 13: 2. Prs. Sg.), nämich die Aussage des Schuldners, daß der Gläubiger keine Ansprüche mehr gegen ihn habe vgl. auch den Dativ beim Verbum in Z. 14 in- $\mathrm{na}-\mathrm{an}-\mathrm{du}_{11}$, der im Zusammenhang mit

$72.13 \mathrm{zu}$ sehen ist).

${ }^{42}$ Zur Formulierung des Auftretens von Zeugen, wie sie hier vorliegt, vgl. A. Falkenstein, NG I, 68f. Anm. 4. Die Präfixkette in-ra- steht möglicherweise für $* / \mathrm{im}$-ra/-; vgl. zum Problem J. Krecher, Or.NS 54 [1985] 143f., allerdings hätten wir dann hier (entgegen ebenda 154: „Im Falle von $-\mathrm{ra}-$ ist mir keine aus $* / \mathrm{im}-\mathrm{ra} /$ - ableitbare Bildung bekannt. ... Offenbar wurde */im-ra/- nicht gebildet.") den ersten Beleg für eine Bildung */im-ra/( $\mathrm{im} /$ - in ventivischer Bedeutung).

$43 \mathrm{Zu} \mathrm{gi-(n)} \mathrm{vgl.} \mathrm{A.} \mathrm{Falkenstein,} \mathrm{NG} \mathrm{III,} \mathrm{114f.} \mathrm{(u.} \mathrm{a.} \mathrm{„fest} \mathrm{machen,} \mathrm{feststellen,} \mathrm{beweisen“);} \mathrm{P.}$

44 Steinkeller, FAOS 17,84 (u. a. "to confirm [a statement, title]").

Zu (lú-)ní-zu(h) ku 4 vgl. zuletzt P. Steinkeller, FAOS 17, $331 \mathrm{f}$. ("to become/to be declared a thief/criminal"); zu (intransitivem) $\mathrm{ku}_{4}$ in vorliegendem Zusammenhang vgl. auch C. Wilcke, ZA 78 [1988] 27 „,zu etwas werden' (= in eine Gruppe/einen Status eintreten ${ }^{*}$. 
$\operatorname{Di}\left[\mathrm{e}^{?}\right.$
$[\mathrm{x}] \ldots$...

Ausgangspunkt für die hier verhandelte Angelegenheit war ein zwischen der Schmied Lugalusar und einem gewissen Ur-Halmu-DU ${ }^{46}$ geschlossener Werkver trag, bei dem es um die Herstellung einer Axt ging. Zu diesem Zweck hatte de Auftraggeber dem Handwerker das dafür notwendige Material zur Verfügung gestellt. Der Schmied war vereinbarungsgemäß auch seiner Lieferverpflichtun:s nachgekommen ${ }^{47}$ jedoch blieb er dem Auftraggeber noch eine Restmenge Metall schuldig, worüber eine Schuldurkunde ausgefertigt wurde. ${ }^{48}$ Wohl in vertragswidriger Weise hat sich dann der als Gläubiger fungierende Auftraggeber des Materials im Hause des Handwerkers gewaltsam bemächtigt und die entsprechende Schuldurkunde „davongetragen“, d. h., er hat die Urkunde, die ihm als Nachweis für seine Ansprüche gegenüber dem Schmied gedient hatte, nicht an den Schuldner ausgehändigt bzw. in dessen Beisein vernichtet. Dies wäre aber die notwendige Rechtsfolge gewesen. Um nun beweisen zu können, das Ur-Halmu-DU unrechtmäßig gehandelt hat und dieser keine Ansprüche mehr gigen ihn geltend machen könne, wurden (vom Gericht) Zeugen für die gewalsame Aneignung des geschuldeten Metalls hinzugezogen. Jedoch haben die Ze t- $^{-}$ gen den (von Lugalusar geschilderten) Tathergang nicht bestätigt. Offensichtlic h

In Z. 21 scheint lú-ní-zu jedoch nicht nur den „Übeltäter" schlechthin zu bezeichnc.1, sondern ist wohl in Richtung "Lügner" zu interpretieren; zu diesem Problem vgl. bere is A. Falkenstein, NG I, 74 Anm. 6; ders., NG II, 140 zu Z. 16; T. Gomi - S. Sato, SNSAT, 25 ("to be condemned as mendacious"). Die Konstruktion der Wendung macht deutlich, d. R die Zeugen als diejenigen genannt sind, die eine falsche Aussage gemacht haben (dal ar unzutreffend $\mathrm{H}$. Limet, in: The Tablet and the Scroll, 142: "ils ont considéré le fondc ir comme un voleur"). Vgl. ähnlich NG II 84 (= ITT III 6539), 12-16; dazu A. Falkenst n, ebenda 139: „Daß die Zeugen wegen einer falschen Behauptung, die nur in lockerem $74-$ sammenhang mit dem Prozeßgegenstand steht, als Lügner überführt wurden, zeigt, $\alpha$ i $\beta$ vor Gericht sehr ins einzelne gehende Verhöre angestellt werden konnten, von denen al er die Urkunden in ihrer lapidaren Kürze nicht berichten“; zum Tatbestand der falschen Z: :ugenaussage vgl. ders., NG I, $131 \mathrm{f}$.; vgl. auch die folgende Anm.

45 Eine Ergänzung ist schwer möglich; in Z. 22 ist vielleicht die Strafsanktion für die Falscha : sssage zu erwarten. Nach CU $\$ 28$ (vgl. zuletzt M. T. Roth, Law Collections from Mesopertmia and Asia Minor, Atlanta 1995, 20) erwartete den "(falschen) Zeugen, der (wohl rum Gericht als unglaubwürdig), zum Lügner gemacht (erwiesen oder erklärt) worden ist" " $i \mathrm{H}$. Petschow, ZSSR 85 [1968] 10) im Rahmen vermögensrechtlicher Streitfälle eine Geldbu!l3e von 15 Sekeln Silber, also „einen allgemein festbezifferten (Straf-)Betrag" (ebenda 10 Añm. 31 ).

46 Vielleicht identisch mit dem gleichnamigen Schreiber und Sohn des „Mästers“ (ku ruš (a) Urnigar; vgl. etwa MVN XVI 1201, Siegel (ŠS 1); Um. 1913, Siegel (ŠS 5); zu ku rušda igl. H. Waetzoldt, in: G. van Driel - Th. J. H. Krispijn - M. Stol (Hrsg.), zikir šumim. Assyriological Studies Presented to F. R. Kraus on the Occasion of his Seventieth Birthday, Leiden 1982, 392: „nach der Funktion mehr ein Verwalter der Mast- und Schlachtviehbestände“; zum Aufgabenbereich des kurušda vgl. ders., RlA IV [1972-1975] 423.

47 Siehe oben Anm. 34.

${ }^{48} \mathrm{Vgl}$. oben Anm. 35 und 38. 
sagten sie damit (wohl nach Untersuchung durch das Gericht) nicht die Wahrheit, so daß sie als Lügner überführt wurden.

Für die hier zu erörtende Problematik von Interesse ist auch die Darlehensurkunde NRVN I 245 aus Nippur, die zusammen mit einem Kupferdarlehen die Zweckgebundenheit einer bestimmten, zusätzlich notierten Kupfermenge im Sinne eines Werkvertrages verbucht:

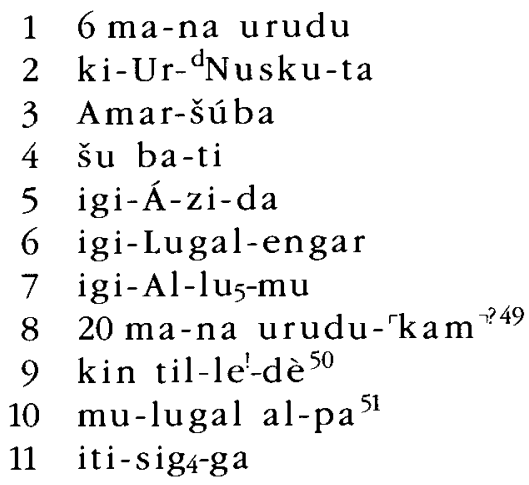

6 Minen Kupfer hat von Ur-Nusku Amaršuba in Empfang genommen. Vor Azida, "or Lugalengar, vor Allumu.

20 Minen Kupfer ${ }^{\ulcorner}$sind $\mathrm{es}^{\urcorner ?}$ ? um das Werk zu vollenden..$^{52}$ Beim König wurde eschworen. Monat: Siga (= III. Monat).“

Nach vorliegender Urkunde erhielt Amaršuba, der hier gewiß als Schmied zu i lentifizieren ist, ${ }^{53}$ von (dem Kaufmann) Ur-Nusku ${ }^{54} 6$ Minen Kupfer als Darlehen, " as durch Zeugen beglaubigt wurde. Darüber hinaus werden 20 Minen Kupfer

Vgl. H. Sauren, ZA 59 [1969] 23.

Vgl. die Kollationsbemerkungen bei H. Waetzoldt, OrAnt. 14 [1975] 312.

Zur unorthographischen Schreibung pa für pà vgl. H. Sauren, ZA 59 [1969] $24 \mathrm{f}$.

Zu kin = šipru „Arbeit(sauftrag) vgl. A. Falkenstein, NG III, 128; E. Sollberger, The Business and Administrative Correspondence under the Kings of Ur, Locust Valley 1966 (TCS I), 142; J. Krecher, in: B. Hruška - G. Komoróczy (Hrsg.), Festschrift Lubor Matouš II, 51 (,Werk"). Zu til = gamäru ${ }_{\text {zu }}$ Ende bringen, vollenden" o. ä. vgl. A. Falkenstein, NG III, 166; E. Sollberger, TCS I, 180; AHw 276b s.v. gamäru(m); M.-L. Thomsen, MCSA 10, 320 (auch zur literarisch belegten Form til-le/e-dè; vgl. dazu jetzt P. Michalowski, The Lamentation over the Destruction of Sumer and Ur, Winona Lake 1989 (MC 1), 38 Z. 47 und 50 ).

Vgl. NRVN I 247, 1 (Dne.); auch hier geht es um eine Angelegenheit zwischen Amaršuba und Ur-Nusku (zu Z. 1-3 des Textes vgl. P. Attinger, Éléments de linguistique sumérienne. La contruction de du $1 /$ e/di $<<$ dire $>>$, Fribourg - Göttingen 1993 [OBO Sonderband], 435).

${ }^{5}$ Zum Kaufmann Ur-Nusku und zu seinen Geschäften vgl. H. Neumann, in: M. deJ. Ellis (Hrsg.), Nippur at the Centennial, Philadelphia 1992 (CRRA 35), 169-173; ders., in: D. Charpin - F. Joannès (Hrsg.), La circulation des biens, des personnes et des idées dans le Proche-Orient ancien, Paris 1992 (CRRA 38), 89. 
genannt, die zur Vollendung eines Werkes bzw. Arbeitsauftrages ohne nähere Charakterisierung dienen sollten.

Das nicht nur im Bereich des (Edel-)Metall- und Holzhandwerks private Aufträge von den Handwerkern angenommen und ausgeführt wurden, ${ }^{55}$ sondern dies auch für andere Handwerkszweige gilt, ist mit Sicherheit anzunehmen. Allerdings sind für jene Bereiche der handwerklichen Produktion, in denen weniger wertvolle Materialien verarbeitet wurden, in weitaus geringerem Maße schriftlich fixierte Werkverträge bzw. damit im Zusammenhang stehende Schuldurkunden zu erwarten. So dürfte z. B. im Rahmen des leder- und rohrverarbeitenden Handwerks bzw. bei den Töpfern für den potentiellen Kunden der Kauf von bereits hergestellten Produkten eine größere Rolle als der vertraglich vereinbarte Arbeitsauftrag gespielt haben. ${ }^{56}$ Grundsätzlich gilt aber daß Handwerker, die für die Palast- und Tempelwirtschaft Auftragsarbeiten ausführten, natürlich erst recht für den privaten Bedarf arbeiten konnten und dies auch taten. ${ }^{57}$

${ }^{55} \mathrm{Vgl}$. in diesem Zusammenhang auch die Kupferdarlehen MVN XI 174 und UET III 37. V. ferner die Darlehensurkunde NRVN I 192 (Zinsantichrese in Form von Zimmermannsa beit); dazu H. Neumann, SGKAO 19, 154 mit Anm. 878.

56 Was die Ausfertigung von Urkunden über den Kauf von handwerklichen Produkten bitrifft, so dürfte dies sicher nicht die Regel, sondern die Ausnahme dargestellt haben. Dre Schriftlichkeit wurde in derartigen Fällen erst dann erforderlich, wenn Leistung und G:genleistung zeitlich auseinanderfielen, also beim Kredit- und Lieferungskauf; vgl. M. San Nicolò. Die Schlußklauseln der altbabylonischen Kauf- und Tauschverträge. Ein Beitrag zur Geschichte des Barkaufs, ${ }^{2} 1974,76 \mathrm{ff}$.

57 Vgl. oben mit Anm. 5-12; vgl. in diesem Zusammenhang auch H. Neumann, CRRA 35 , 166f. mit Anm. 31 (korrigiere die Bemerkung zu TMH NF I/II 55: Vertragsparteien versehentlich vertauscht; zur Urkunde vgl. auch J.-M. Durand, RA 71 [1977] 135 Anm. 4) zur Problematik privater handwerklicher Produktion im Bereich der Lederverarbeitung. Zur Ausführung privater Bauaufträge in der Ur III-Zeit vgl. H. Neumann, Der sumerische Batmeister (šidim), in: Houses and Households in Ancient Mesopotamia, Istanbul (CRRA 40) [im Druck]. 